\title{
El cambio en la estructura de la industria de confección en Colombia, la subcontratación y el desarrollo de proveedores ${ }^{1}$
}

The change in the structure of the garment industry in Colombia, outsourcing and supplier development

Recibido: 29 de septiembre de 2015 - Revisado: 16 de noviembre de 2015 - Aceptado: 12 de enero de 2016

Jorge Eduardo Medina Fernández de Soto ${ }^{2}$

\section{Resumen}

La estructura de la industria de la confección está cambiando en el mundo: la distribución y comercialización se está realizando a través de grandes cadenas de comercialización, multinacionales con marcas propias e hipermercados, las cuales utilizan la subcontratación para responder a las exigencias de los clientes finales en precios, diseño, velocidad de entrega y calidad. Este cambio ha afectado el sector textil manufacturero en Colombia, el cual debe estar preparado para identificar y consolidar empresas líderes y aumentar su competitividad. En este artículo se busca definir los criterios para la selección y el desarrollo de la subcontratación, teniendo en cuenta que existen dos tipos básicos: las maquiladoras y los de paquete completo.

\section{Palabras clave}

Subcontratación, producción y operaciones, cadena de suministro, valor agregado.

\begin{abstract}
The structure of the garment industry is changing in the world: the distribution and marketing is being done through large marketing chains, multinational companies with own brands and hypermarkets, which use outsourcing to meet the demands of end customers in price, design, delivery speed and quality. This change has affected the textile manufacturing sector in Colombia, which must be prepared to identify and consolidate leading companies and increase their competitiveness. This article seeks to define the criteria for the selection and development of outsourcing, considering that there are two basic types: the maquila industry and the full package one.
\end{abstract}

\section{Keywords}

Outsourcing, production and operations, supply chain, added value.

\footnotetext{
${ }^{1}$ Artículo de investigación desarrollado en el marco del proyecto "Modelo de desarrollo de proveedores para industria de la confección" de la Universidad Sergio Arboleda, Bogotá, Colombia.

2 Magíster en Docencia e Investigación Universitaria de la Universidad Sergio Arboleda, Bogotá, Colombia. Especialista en Administración de Negocios de la misma institución. Ingeniero industrial de la Pontificia Universidad Javeriana, Bogotá, Colombia. Director de la Maestría en Producción y Operaciones. Director de las especializaciones: Gerencia de Producción y Operaciones; y Gerencia Integral de la Calidad. Docente investigador. Escuela de Ciencias Exactas e Ingeniería, Universidad Sergio Arboleda. Correo electrónico: jorge.medina@usa.edu.co

Para citar este artículo use: Medina, J. (2016). El cambio en la estructura de la industria de confección en Colombia, la subcontratación y el desarrollo de proveedores. Revista Civilizar Ciencias Sociales y Humanas, 16(30), 137-144.
} 


\section{Introducción}

La industria de confecciones está cambiando su estructura para competir en los mercados mundiales, para ello requiere el desarrollo de empresas líderes, susceptibles de convertirse en firmas de clase mundial, con capacidad de diseño y comercialización que subcontratan toda o parte de la manufactura. Estas empresas líderes son las que tienen posibilidades de generar estrategias para competir internacionalmente o convertirse a su vez en proveedoras para las grandes cadenas de comercialización internacional de prendas de vestir, cuya tendencia es comprar productos terminados de proveedores en diferentes países (Ananth, Seshadri, \& Vasher, 2010).

Para analizar este cambio se puede observar el caso de España, en donde las empresas de confecciones no pudieron competir en segmentos de bajo costo debido a la ausencia de economías de escala y los altos gastos financieros. España tiene similitudes con Colombia al ser un importador de materias primas como el algodón, lo que ocasiona que los productos finales resulten más costosos comparativamente con los países que poseen estas materias primas como recurso natural.

Es importante observar que al trasladarse la distribución y comercialización internacional a las grandes cadenas de comercialización, multinacionales con marca propia e hipermercados, se genera un incremento en la utilización de la subcontratación como estrategia de abastecimiento de estas empresas con diversos modelos de proveedores que van desde el empleo de maquiladoras, hasta servirse de proveedores de paquete completo.

Además, los productos comercializados están siendo cada vez más sofisticados (con creatividad y moda), de mayor valor agregado (con innovación en materiales y diseños) y de bajo costo, por lo que la industria colombiana debe identificar empresas líderes que sean capaces de competir nacional e internacionalmente y afrontar la competencia de las cadenas internacionales, las multinacionales con marca propia y las ventas de ropa a través de hipermercados.

En el caso español el fortalecimiento de los nuevos sistemas de distribución y comercialización, se puede evidenciar no solo en la presencia de las grandes cadenas de comercialización internacional en el mercado, sino en el crecimiento y desarrollo de multinacionales con marca propia (Costa \& Duch, 2010). Esta transformación ha ocasionado el surgimiento en España de multinacionales capaces de competir internacionalmente, la presencia en su territorio de grandes cadenas de comercialización e hipermercados extranjeros y el incremento de la subcontratación en la industria de la confección.

Para analizar las características del sistema de gestión y desarrollo de proveedores en el sector moda, se tomó como objeto de estudio a la empresa española Zara ${ }^{\circledR}$ del grupo Inditex, por ser la de más rápido crecimiento en el mundo y por sus estrategias novedosas y efectivas, con resultados contundentes. Las plantas existentes no producen en su totalidad, se consideran parte de cadenas ensambladoras con la particularidad de que en ellas se inicia y termina el ciclo de fábrica. El proceso arranca por el corte de las piezas de tela, a partir de las creaciones de la red de diseño. De ello resultan las piezas identificadas en posición, prenda y talla, acompañadas de botones, cremalleras y demás complementos, los cuales se introducen en bolsas para ser remitidos a los talleres satélite donde se realizará la costura y terminado de las prendas. En lugar de la fabricación masiva de cada modelo, el proceso productivo se adapta a las peticiones de las tiendas y, por tanto, a la demanda del mercado.

Para la elección de los proveedores la empresa aplica un proceso de cotización del 
trabajo entre diferentes talleres, buscando la velocidad en la entrega y negociando el costo de este trabajo con criterios de productividad (Organización de las Naciones Unidas para el Desarrollo Industrial, s.f.).

La relación con los proveedores ha pasado de un simple intercambio comercial al trabajo como socios estratégicos y a los criterios tradicionales de compra (precio, condiciones de pago y entregas a tiempo) se les han sumado otros como flexibilidad, trabajo colaborativo y aseguramiento de la calidad. Este modelo se orienta a la conformación de una "cadena de suministro" que es la red de servicios, materiales y flujos de información que vincula los procesos de relación con los clientes, surtido de pedidos y proveedores de una empresa, con los procesos de sus proveedores y clientes (Krajewski, Ritzman, \& Malhotra, 2008).

\section{Problema de investigación}

Colombia se ve abocada a una transformación similar, desarrollando empresas líderes que puedan competir internacionalmente y que utilicen la capacidad de producción de sus proveedores para aumentar la flexibilidad para producir diferentes modelos y acrecentar la velocidad en los tiempos de entrega al cliente final, utilizando capacidad de producción externa y de esta manera disminuyendo la inversión para ampliar la capacidad interna (Gómez, 1996).

El cambio estructural que implica la puesta en marcha de empresas líderes conlleva la subcontratación de empresas proveedoras capaces de responder a las exigencias de productividad, calidad, innovación y velocidad de entrega; por lo cual la investigación busca la determinación de los criterios para seleccionar y desarrollar los tipos de proveedores adecuados a partir del análisis de maquiladoras y proveedores de paquete completo.
El análisis parte de la identificación de las características de los proveedores y de los procesos relacionados, para plantear posteriormente el modelo de selección adecuado, los criterios y los indicadores de resultado y de desempeño de cada uno.

\section{Tipos de proveedores.}

Existen básicamente dos tipos de proveedores de manufactura de confección:

Proveedores tipo 1: maquiladoras, se les proporcionan las materias primas, diseño de las prendas, patrones de confección y las piezas cortadas. Estos satélites se encargan de realizar la confección de las prendas hasta el embalaje, según parámetros establecidos previamente en un acuerdo y que deben ir de la mano con el sistema integral de aseguramiento de calidad de la compañía líder.

Proveedores tipo 2: paquete completo, colocan materia prima e insumos, realizan los procesos de confección y terminado y pueden elaborar el diseño cuando la empresa líder necesite generar diseños adicionales para satisfacer la demanda de los clientes finales, cuentan con abastecimiento de materias primas e insumos certificados, sistemas de producción eficaces y control de calidad, así como la logística de distribución de producto terminado para garantizar la colocación del producto en los puntos de venta del cliente final de manera oportuna.

Es muy importante tener la estructura de costos de la industria ya que la materia prima constituye un $36 \%$, la mano de obra un $29 \%$ y otros un $35 \%$. Por lo que la estrategia de las firmas líderes puede orientarse a la contratación de la mano de obra en confección y terminado, haciendo la compra directa de las materias primas y suministrándolas al proveedor. La inclusión del diseño puede ser opcional, según las necesidades de la empresa líder (Ministerio de Comercio, Industria y Turismo, 2009). 
Gráfica 1

Sistema de producción de una empresa líder, maquiladoras y de paquete completo

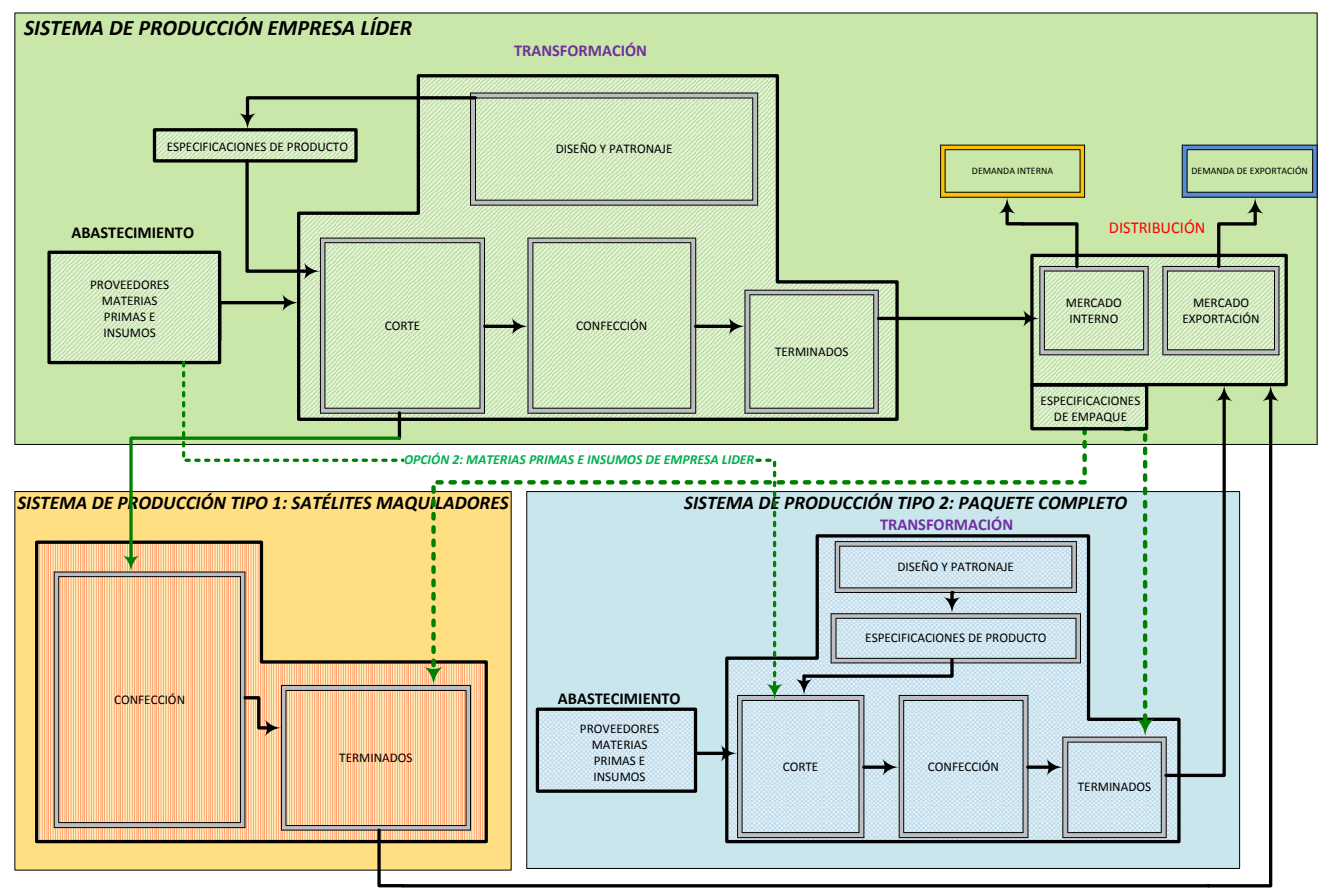

Fuente: elaboración propia.

\section{Metodología}

Para analizar el desarrollo de los proveedores en Colombia se diseñó un estudio de caso, para ello se escogió una empresa líder que trabaja con proveedores externos y se realizó una evaluación a cuatro de ellos (dos de paquete completo y dos maquiladoras). A continuación se exponen los aspectos y criterios empleados. En cada proveedor se tuvieron en cuenta los factores que muestra la tabla 1.

\section{Tabla 1}

\section{Factores de formalización}

\begin{tabular}{|ll}
1 & Gestión estratégica \\
\hline 2 & Producción \\
3 & Logística \\
4 & Calidad \\
5 & Diseño \\
6 & Factor organizacional \\
\hline
\end{tabular}

Fuente: elaboración propia.

Los factores de formalización no fueron calificados cuantitativamente, estos se anali- zaron evidenciando su existencia. Dentro de los criterios de formalización, los clasificados como críticos se indican enseguida, estos deben siempre existir para que el proveedor pueda comenzar a trabajar con la empresa.

- Disponibilidad de los recursos financieros suficientes para desarrollar el plan estratégico por parte del proveedor.

- Empresa legalmente constituida (registro de cámara de comercio).

- Pago de prestaciones sociales para los diferentes tipos de contratos.

- Definición de organigrama y manual de funciones y responsabilidades.

El estudio arroja que las empresas mejor calificadas en todos los factores son las de paquete completo. Las diferencias más significativas se pudieron observar en los procesos de gestión estratégica, diseño y logística (véase tabla 2). 
Tabla 2

Calificación de factores

\begin{tabular}{|c|c|c|c|c|c|}
\hline & & \multicolumn{2}{|c|}{ Paquete completo } & \multicolumn{2}{|c|}{ Maquiladora confección } \\
\hline \multicolumn{2}{|c|}{ Factores de formalización } & $\begin{array}{c}\text { Proveedor } 1 \\
(\%)\end{array}$ & $\begin{array}{c}\text { Proveedor } 2 \\
(\%)\end{array}$ & $\begin{array}{c}\text { Proveedor } 3 \\
(\%)\end{array}$ & $\begin{array}{c}\text { Proveedor } 4 \\
(\%)\end{array}$ \\
\hline 1 & Gestión estratégica & 100 & 100 & 0 & 0 \\
\hline 2 & Producción & 95,24 & 88,89 & 55,57 & 79,37 \\
\hline 3 & Logística & 81,03 & 100 & 0 & 20,69 \\
\hline 4 & Calidad & 96,51 & 91,86 & 48,81 & 51,13 \\
\hline 5 & Diseño & 100 & 80 & 0 & 0 \\
\hline 6 & Factor organizacional & 97,10 & 94,93 & 80,44 & 47,12 \\
\hline
\end{tabular}

Fuente: elaboración propia.

Las maquiladoras no trabajan con metodología estratégica ni planeación financiera. Son empresas reactivas que tienden a depender de un solo cliente y cuya sostenibilidad se sujeta a producir volumen con una baja rentabilidad por su trabajo. En cuanto a diseño las empresas de paquete completo lo ofrecen y las maquiladoras no.

Como consecuencia de no tener los recursos financieros suficientes, las maquiladoras presentan deficiencias en la gestión del transporte y en los sistemas de almacenamiento y distribución.

Las empresas de paquete completo reportan fortalezas en sus sistemas de producción: la programación de la producción por lotes con base en los pedidos de los clientes y utilización de alta tecnología en corte y confección.

Los proveedores de paquete completo tienen la calidad como pilar para el logro de la satisfacción del cliente, lo que ayuda a su posicionamiento en el mercado y por consiguiente al aumento de la rentabilidad. Además manejan políticas de selección, estructura salarial, higiene y seguridad industrial, bienestar, clima organizacional, estructura organizacional y capacitación. Son también empresas legalmente constituidas.

En cuanto a los factores de formalización las cuatro empresas cumplen los aspectos críticos. En general los proveedores de paquete completo cumplen la mayoría de los aspectos analizados, como puntos débiles están la falta del mejoramiento de condiciones de trabajo.

\section{Análisis y definición de criterios de selección y desarrollo de los proveedores}

Se definieron tres grandes aspectos: dirección estratégica, organización y proceso de formalización de la compañía. A continuación se especifican los criterios y los indicadores de desempeño y de resultado.

Los criterios de dirección estratégica buscan que los proveedores desarrollen su negocio y cuenten con los recursos para la consecución de una empresa sostenible (véase tabla 3). 
Tabla 3

Criterios de dirección estratégica

\begin{tabular}{|c|c|c|c|}
\hline & Criterio & Indicador de resultados & Indicador de desempeño \\
\hline \multirow{4}{*}{ 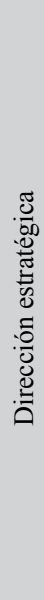 } & $\begin{array}{l}\text { Importancia del nego- } \\
\text { cio de la empresa líder } \\
\text { dentro del negocio total } \\
\text { del proveedor }\end{array}$ & $\begin{array}{l}\text { Consolidación de su negocio como proveedor } \\
\text { de la empresa líder }\end{array}$ & $\begin{array}{l}\text { Participación como proveedor de la organización } \\
\text { líder dentro del negocio total de la empresa líder }\end{array}$ \\
\hline & $\begin{array}{l}\text { Plan estratégico que re- } \\
\text { fleje un desarrollo de la } \\
\text { empresa }\end{array}$ & $\begin{array}{l}\text { Existencia e implementación de un plan estra- } \\
\text { tégico con objetivos, metas y recursos }\end{array}$ & $\begin{array}{l}\text { Existencia e implementación de un plan estraté- } \\
\text { gico completo }\end{array}$ \\
\hline & \multirow{2}{*}{$\begin{array}{l}\text { Plan financiero que ga- } \\
\text { rantice los recursos, las } \\
\text { inversiones necesarias } \\
\text { para desarrollar el plan } \\
\text { estratégico y un creci- } \\
\text { miento sostenible }\end{array}$} & \multirow{2}{*}{$\begin{array}{l}\text { Disponibilidad de los recursos financieros su- } \\
\text { ficientes para desarrollar el plan estratégico }\end{array}$} & $\begin{array}{l}\text { Presupuesto de ingresos y egresos que garantice } \\
\text { los recursos suficientes. "Proyección de la produc- } \\
\text { tividad integral" }\end{array}$ \\
\hline & & & $\begin{array}{l}\text { Indicadores financieros y otros (liquidez, endeu- } \\
\text { damiento, capital de trabajo y prueba ácida) }\end{array}$ \\
\hline
\end{tabular}

Fuente: elaboración propia.

Los criterios de organización buscan trabajar con empresas legalmente constituidas y que cumplan con las políticas de bienestar exigidas en la ley.

Tabla 4

Criterios de organización

\begin{tabular}{|c|c|c|c|}
\hline & Criterio & Indicador de resultados & Indicador de desempeño \\
\hline \multirow{6}{*}{ 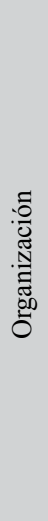 } & Empresa legalmente constituida & $\begin{array}{l}\text { Registro de la empresa en la cámara de } \\
\text { comercio }\end{array}$ & Certificado de cámara de comercio y RUT \\
\hline & $\begin{array}{l}\text { Tiempo de constitución de la } \\
\text { empresa }\end{array}$ & $\begin{array}{l}\text { Registro de la empresa en la cámara de } \\
\text { comercio }\end{array}$ & Certificado de cámara de comercio y RUT \\
\hline & \multirow{3}{*}{ Bienestar del personal } & \multirow{3}{*}{$\begin{array}{l}\text { Pago de prestaciones sociales } \\
\text { Tipo de contratación del personal }\end{array}$} & $\begin{array}{l}\text { Pago de EPS, ARL, pensiones, cesantías y } \\
\text { caja de compensación familiar }\end{array}$ \\
\hline & & & Personal indefinido, temporal, por servicios \\
\hline & & & Rotación del personal \\
\hline & $\begin{array}{l}\text { Definición de funciones y res- } \\
\text { ponsabilidades }\end{array}$ & $\begin{array}{l}\text { Definición de organigrama y manual } \\
\text { de funciones y responsabilidades }\end{array}$ & $\begin{array}{l}\text { Cargos establecidos y con manual de funcio- } \\
\text { nes y responsabilidades }\end{array}$ \\
\hline
\end{tabular}

Fuente: elaboración propia.

Los criterios de transformación buscan que los proveedores posean procesos desarrollados con indicadores claros y metodologías adecuadas para poder dar cumplimiento a los requerimientos de los clientes. 
Tabla 5

Criterios de transformación

\begin{tabular}{|c|c|c|c|}
\hline & Criterio & Indicador de resultados & Indicador de desempeño \\
\hline \multirow{17}{*}{ 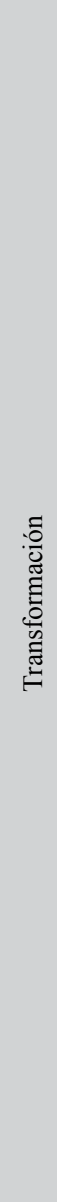 } & \multirow{5}{*}{ Capacidad de producción } & \multirow{4}{*}{$\begin{array}{l}\text { Cantidad de productos en un periodo } \\
\text { de tiempo }\end{array}$} & Capacidad de producción \\
\hline & & & $\begin{array}{l}\text { Eficiencia dentro de cada uno de los recursos involu- } \\
\text { crados }\end{array}$ \\
\hline & & & Cantidad producida sobre cantidad total programada \\
\hline & & & Maquinaria de última tecnología, uso y mantenimiento \\
\hline & & Entrega a tiempo & Entregas oportunas sobre entregas totales \\
\hline & \multirow{4}{*}{$\begin{array}{l}\text { Calidad en el producto } \\
\text { terminado }\end{array}$} & \multirow{4}{*}{$\begin{array}{l}\text { Cumplimiento de todos los estánda- } \\
\text { res de calidad establecidos }\end{array}$} & $\begin{array}{l}\text { Calidad del producto (características de calidad/acuer- } \\
\text { do de calidad) }\end{array}$ \\
\hline & & & Concesiones y reprocesos \\
\hline & & & Servicio \\
\hline & & & Embalaje \\
\hline & \multirow{4}{*}{ Flexibilidad } & \multirow{4}{*}{$\begin{array}{l}\text { Respuesta a las variaciones en canti- } \\
\text { dades solicitadas }\end{array}$} & $\begin{array}{l}\text { Capacidad de aceptar órdenes de compra nuevas sobre } \\
\text { la marcha sin afectar el cumplimiento de las demás } \\
\text { órdenes de compra }\end{array}$ \\
\hline & & & $\begin{array}{l}\text { Capacidad de aumentar la cantidad del pedido inicial } \\
\text { sobre la marcha }\end{array}$ \\
\hline & & & Capacidad de turnos adicionales \\
\hline & & & $\begin{array}{l}\text { Capacidad de producción disponible para la empresa } \\
\text { líder (reserva) }\end{array}$ \\
\hline & \multirow{3}{*}{$\begin{array}{l}\text { Grado de innovación del } \\
\text { producto }\end{array}$} & \multirow{3}{*}{ Innovación del producto } & Existencia del departamento de diseño \\
\hline & & & Capacidad de generar nuevos diseños por semana \\
\hline & & & Aceptación de los diseños (originalidad y buen gusto) \\
\hline & $\begin{array}{l}\text { Productividad en el proceso } \\
\text { de transformación }\end{array}$ & $\begin{array}{l}\text { Nivel de productividad mínimo } \\
\text { aceptable }\end{array}$ & $\begin{array}{l}\text { Productividad del proceso de transformación y de cada } \\
\text { recurso }\end{array}$ \\
\hline
\end{tabular}

Fuente: elaboración propia.

\section{Calidad.}

Dada la importancia de la calidad del producto confeccionado por el proveedor para determinar su selección y su desarrollo, se debe incluir acuerdos de calidad, los cuales se consideran necesarios para que el proveedor desarrolle sistemas de aseguramiento de calidad.

El aseguramiento de calidad se define como el conjunto de actividades planificadas y metódicas necesarias para proporcionar la confianza apropiada de que un producto o servicio satisface los requisitos de calidad y de esta forma dar cumplimiento a las expectativas del cliente. El sistema de aseguramiento se traduce en "acuerdos de calidad" que deben ser cumplidos por el proveedor, para alcanzar la calidad requerida por el cliente final.

Aunque el sistema de aseguramiento pretende garantizar niveles de calidad de "cero defectos", la empresa líder debe conservar el control sobre las quejas, requerimientos y reclamos del cliente, para poder identificar casos de fallas (Medina \& Cordero, 2010). 


\section{Productividad.}

La gestión de productividad debe orientarse al mejoramiento de los estándares de la empresa líder en los diferentes proveedores, teniendo en cuenta que el control para las maquiladoras estará sobre la cantidad y calidad del trabajo de confección y terminado, mientras que para paquete completo incluirá procesos adicionales como el diseño y la entrega.

Pero el modelo de negociación con los proveedores debe tener en cuenta que en el caso del proveedor tipo 1 (maquiladoras), debe considerar básicamente el traslado de los costos de mano de obra y las prestaciones sociales a un proveedor externo (el cual además debe generar márgenes de utilidad para subsistir como proveedor y asumir costos indirectos de fabricación), este debe por lo tanto mantener altos niveles de productividad laboral. En el caso del proveedor de paquete completo, se debe considerar que este asumirá no solamente costos de mano de obra y prestaciones sociales, sino también el mantenimiento de un área de diseño. Además si el proveedor proporciona las materias primas e insumos se debe controlar la productividad de las materias primas por prenda terminada (Medina, 2007).

\section{Conclusiones y recomendaciones}

El modelo propuesto para la selección de proveedores permitirá a la empresa líder tener una guía detallada y clara acerca de cómo gestionar a sus proveedores de confecciones, con el fin de garantizar el óptimo funcionamiento de la cadena de abastecimiento, hasta la entrega del producto final al cliente cumpliendo todos los requerimientos.

Las evaluaciones de proveedores implementadas como un programa estratégico de la compañía, lejos de ser un costo impuesto para satisfacer un requisito formal, constituyen una sólida inversión destinada a obtener una excelente relación con los proveedores buscando alta productividad, calidad, velocidad en la entrega y competitividad.

El compromiso con el sistema de aseguramiento de la calidad y la productividad por parte de los proveedores debe formar parte de la escogencia y definición de los mismos.

\section{Referencias}

Ananth, I., Seshadri, S., \& Vasher, R. (2010). Administración de la cadena de suministro Toyota. México: Mc Grow Hill.

Costa, M. T., \& Duch, N. (2010). La renovación del sector textil- confección en España. Barcelona: Instituto de economía de Barcelona.

Gómez, E. (1996). Aseguramiento de calidad en compras. Bogotá: RAM Editores.

Krajewski, L. J., Ritzman, L. P., \& Malhotra, M. K. (2008). Administración de operaciones. Procesos y cadenas de valor. Mexico: Pearson Educación.

Medina, J. (2007). Modelo Integral de la productividad. Bogotá: Fondo de publicaciones Universidad Sergio Arboleda.

Medina, J., \& Cordero, N. (2010). Gestión Estratégica de la Calidad. Bogotá: Fondo de publicaciones Universidad Sergio Arboleda.

Ministerio de Comercio, Industria y Turismo. (2009). Desarrollando sectores de clase mundial en Colombia. Bogotá: Autor.

Organización de las Naciones Unidas para el Desarrollo Industrial. (S.f.). Guía para el Desarrollo de Proveedores. Recuperado de https://www.unido.org/fileadmin/import/9605_GuaparelDesarrollodeProveedores.pdf 